\title{
Sclerosing TFEB Rearrangement Renal Cell Carcinoma: A Recurring Histologic
}

\section{Pattern}

Sean R Williamson, $\mathrm{MD}^{1,2,3}$; John N Eble, MD, MBA ${ }^{4}$; Nallasivam Palanisamy, $\mathrm{PhD}^{5,6}$

${ }^{1}$ Department of Pathology and Laboratory Medicine, and ${ }^{2}$ Josephine Ford Cancer Institute, Henry Ford Health System, Detroit, MI, USA; ${ }^{3}$ Wayne State University School of Medicine, Detroit, MI, USA; ${ }^{4}$ Department of Pathology and Laboratory Medicine, Indiana University School of Medicine, Indianapolis, IN, USA; ${ }^{5}$ Department of Urology, Vattikutti Urology Institute, Henry Ford Health System, Detroit, MI, USA; ${ }^{6}$ Michigan Center for Translational Pathology, University of Michigan, Ann Arbor, MI, USA.

Address correspondence and reprint requests to:

Sean R. Williamson, MD

Henry Ford Hospital, Department of Pathology - K6

2799 West Grand Blvd

Detroit, MI 48202

Email: seanwill@temple.edu

Phone: 313-916-3986

Fax: 313-916-2385

Conflicts of Interest and Source of Funding: None declared

Key words: renal cell carcinoma, $T F E B$ gene, translocation, immunohistochemistry, fluorescence in situ hybridization

This is the author's manuscript of the article published in final edited form as:

Williamson, S. R., Eble, J. N., \& Palanisamy, N. (2017). Sclerosing TFEB-rearrangement renal cell carcinoma: a recurring histologic pattern. Human Pathology, 62, 175-179. https://doi.org/10.1016/j.humpath.2016.10.024 


\section{INTRODUCTION}

Renal cell carcinoma with $T F E B$ gene rearrangement was originally recognized based on translocation $t(6 ; 11)$ occurring in children.[1] The unique histologic appearance of these tumors was first characterized as including large cells with clear or eosinophilic cytoplasm and a second population of smaller cells with scant cytoplasm arranged around hyaline basement membrane material, resembling rosettes.[1,2] More recently, however, other studies have found that this unique morphology is not always straightforward, and that some molecularly-confirmed $T F E B$ rearrangement tumors may mimic clear cell renal cell carcinoma, chromophobe renal cell carcinoma, tubulocystic renal cell carcinoma, epithelioid angiomyolipoma, multilocular cystic renal cell carcinoma, or high-grade unclassified renal cell carcinoma.[3-6] One recent series included a single example that was extensively hyalinized and ossified.[4] Herein we report another such example with extensive sclerosis and metaplastic bone formation, nearly obscuring the epithelial nature of the tumor, suggesting that this tendency for sclerosis may be a recurring pattern of TFEB renal cell carcinoma.

\section{MATERIALS AND METHODS}

\section{Immunohistochemistry}

Antibodies directed against $\alpha$-methylacyl-coA-racemase (AMACR/P504S (13H4; Dako Corp, Carpinteria, CA, USA), carbonic anhydrase IX (polyclonal rabbit; Abcam, Cambridge, UK), cathepsin K (3F9; Abcam), CD10 (56C6; Dako Corp), cytokeratin (AE1/AE3; Dako Corp), cytokeratin 7 (CK7) (OV-TL 12/30; Dako Corp), caldesmon (hCD; Dako Corp), epithelial membrane antigen (EMA; E29; Dako), human melanosome (HMB45; Dako Corp), inhibin (R1; Dako Corp), melan-A (A103; Dako Corp), PAX8 
(polyclonal; Cell Marque, Rocklin, CA, USA), smooth muscle actin (1A4 clone; Dako Corp), TFE3 (MRQ-37; Cell Marque), and vimentin (V9; Dako Corp) were utilized in a Dako automated instrument. Positive and negative controls yielded appropriate results for each antibody.

Fluorescence in situ hybridization (FISH)

Bacterial artificial chromosome (BAC) clones from the immediately flanking genomic regions of TFEB and TFE3 genes were used to generate dual color break-apart FISH probes (TFEB: RP11-246A15- 5', RP11-624F22-3'; TFE3: RP11-528A24,-5', RP11-416B14-3'). BAC clones were tested on normal human metaphase chromosomes to validate map position. Probes were detected with anti-digoxigenin fluorescein Fab fragments to yield green color and Streptavidin Alexa fluor 594 to yield red color for the 5' and 3' probes respectively. Overnight $200 \mathrm{ml}$ cultures for each BAC clone were grown in LB medium containing $12.5 \mu \mathrm{g} / \mathrm{ml}$ of chloramphenicol at $37^{\circ} \mathrm{C}$ for $14-16$ hours with constant shaking. DNA was prepared using Qiagen-midiprep kit using Qiatip-100 according to the protocol provided by the manufacturer (Qiagen, Hilden, Germany).

All FISH probes were prepared by nick translation labeling using modified nucleotides conjugated with biotin or digoxigenin utilizing biotin nick translation mix (11745824910, Roche, Basel, Switzerland) and digoxigenin nick translation mix (11745816910, Roche) for 3' and 5' probes respectively. Probe DNA was precipitated and dissolved in hybridization mixture containing $50 \%$ formamide, 2 XSSC, $10 \%$ dextran sulphate, and 1\% Denhardt's solution. Approximately 200ng of each labeled probe was used for hybridization. Fluorescent signals were detected with Streptavidin Alexa fluor 594 
(S-32356, Invitrogen, Carlsbad, CA, USA) and anti-digoxigenin fluorescein Fab fragments (11207741910, Roche) for red and green colors, respectively.

\section{CASE REPORT}

A 54 year-old woman found to have an endophytic, enhancing renal mass in the right kidney (Figure 1A). Partial nephrectomy was performed, and the specimen was evaluated by two of the authors (SRW and JNE) for classification of the extensively sclerotic and calcified renal mass, measuring 2.4 x $1.5 \times 1.5 \mathrm{~cm}$. Histologic sections revealed predominant fibrosis, hyalinization, calcification and ossification (Figure 1B). Adipose tissue was focally present, adjacent to the areas of bone formation, likely representing metaplastic formation of bone marrow. Occasional thick-walled blood vessels were also present (Figure 1B), raising consideration of an unusual pattern of angiomyolipoma. A minority of the neoplasm was composed of aggregates of epithelioid cells with variably clear to eosinophilic, vacuolated cytoplasm forming solid sheets and focally cysts (Figure 1C-D). The nuclei of these cells were round and uniform, although with focally moderate variability in nuclear size (Figure 1D). Rare cells revealed multiple nuclei arranged around a central eosinophilic area, with peripheral vacuolization of the cytoplasm, similar to epithelioid cells that have been reported in angiomyolipoma. Based on these findings, immunohistochemical staining to evaluate a differential diagnosis including sclerotic renal cell carcinoma, angiomyolipoma, and renal hemangioblastoma was undertaken.

Immunohistochemical staining revealed the epithelioid cells to be positive diffusely for cytokeratin AE1/AE3 (Figure 2A, 90\% of recognizable epithelioid cells) and PAX8 (Figure 2B, at least 90\% of recognizable epithelioid cells) and negative for caldesmon, 
SMA, TFE3 protein, carbonic anhydrase IX, CD10, CK7, EMA and inhibin. Variable labeling for melan-A (Figure 2C, 5-10\% of cells) and cathepsin K (Figure 2D, 50-60\% of cells) was present in the epithelioid component, and antibodies to HMB-45 (Figure 2E) and AMACR (p504s) labeled rare cells (1\% of cells or less). Fluorescence in situ hybridization revealed rearrangement (split signals) for TFEB (Figure 2F) and no rearrangement of TFE3.

\section{DISCUSSION}

Renal cell carcinoma with TFEB rearrangement is now regarded as part of a group of related tumors with Xp11.2 translocation carcinoma (TFE3 translocation renal cell carcinoma), together considered the microphthalmia transcription factor (MITF) rearrangement family of tumors. [7,8] Although initially recognized based on distinctive histology (biphasic cell populations with large clear or eosinophilic cells and small cells with scant cytoplasm and hyaline material) in children,[1] it has been more recently shown that TFEB rearrangement-associated renal cell carcinoma also occurs in adults and that some tumors may not exhibit the prototypical biphasic cell populations, mimicking other subtypes of renal cell carcinoma.[3-6]

Deceptive patterns of TFEB translocation renal cell carcinoma that have been recently recognized include examples with multilocular cystic,[3] oncocytic,[4] eosinophilic papillary,[4] high-grade unclassified,[3,5] chromophobe renal cell carcinomalike,[5] tubulocystic,[6] epithelioid angiomyolipoma-like,[5] or clear cell-like[1,5] features. One example from a series of 8 tumors reported by Argani and colleagues was noted to be extensively sclerotic and ossified,[4] occurring in a 37 year-old man, similar to 
the case reported here. Both in the report by Argani et al and the current case, the tumors yielded labeling for PAX8, melan-A, cathepsin K, and a lesser extent of labeling for HMB45, without the appreciable dual cell population pattern that is characteristic of TFEB translocation-associated tumors.[4] Thus, it appears that an extensively sclerotic histologic pattern, although likely less common than the prototypical appearance, potentially represents a recurring histology of $T F E B$ rearrangement-associated renal cell carcinoma. Since this differs from the distinctive morphology originally reported, pathologists should be aware of this unusual phenomenon to facilitate diagnostic classification.

In this case, we were prompted to consider several differential diagnostic possibilities based on the unusual tumor histology: Clear cell renal cell carcinoma is the most common subtype of renal cell carcinoma, and therefore presumably the most likely to undergo extensive degenerative changes. In the current case, negative reactivity for carbonic anhydrase IX, combined with positive labeling for melanocytic markers (HMB45 and melan-A) and cathepsin K[9] argued against a diagnosis of clear cell renal cell carcinoma and argued in favor of a translocation-associated tumor, which was confirmed by FISH. Due to the mixture of epithelioid clear to eosinophilic cells, thick blood vessels, and fat, we also considered the possibility of an unusual manifestation of angiomyolipoma. Calcification associated with fat in a renal mass is generally considered presumptive evidence for renal cell carcinoma radiographically,[10,11] most likely representing bone marrow fat associated with metaplastic bone.[10,12] However, rare calcification in angiomyolipoma has been reported.[10] Despite that positivity for melanocytic markers and cathepsin $\mathrm{K}$ would be a shared feature of both TFEB translocation renal cell carcinoma and angiomyolipoma, the tumor cells in this case were negative for smooth muscle 
markers (smooth muscle actin, caldesmon) and positive for epithelial markers (keratin AE1/AE3 and PAX8), resolving this distinction. Finally, we also considered the possibility of hemangioblastoma, based on the composition by foamy-appearing cells with clear to eosinophilic cytoplasm. Although much more common in the central nervous system, hemangioblastoma has recently been increasingly recognized to occur as a primary renal tumor, and several studies have recently found unexpected aberrant positivity for PAX8.[13-15] Although few primary renal hemangioblastomas have been thoroughly characterized with a broad immunohistochemical panel, these tumors appear to be consistently positive for inhibin, and negative for melanocytic markers and cathepsin $\mathrm{K}$, and predominantly negative for epithelial markers other than PAX8.[13-16] Interestingly, PAX2 and PAX8 have been found to show negative staining in central nervous system hemangioblastomas, supporting a role as diagnostic markers to distinguish hemangioblastoma from metastatic renal cell carcinoma, both of which are prone to occur in von Hippel-Lindau disease patients.[17] The aberrant PAX8 positivity in primary kidney hemangioblastomas has therefore been hypothesized to reflect origin from an organ-specific cell type through unknown mechanisms.[13,15] Conversely, inhibin positivity has also been reported in some renal cell carcinomas, blurring the distinction between renal cell carcinoma and hemangioblastoma of the kidney in some cases.[18,19] In summary, here we report an example of extensively sclerotic and ossified renal cell carcinoma with $T F E B$ rearrangement, which showed positive immunohistochemical labeling for keratin AE1/AE3 and PAX8, patchy labeling for melan-A and cathepsin K, and very limited labeling for HMB45. Combined with one prior such example from a series of TFEB rearrangement renal cell carcinomas, it appears that this may be a less 
common but recurring histologic pattern of TFEB renal cell carcinoma, which can be distinguished from angiomyolipoma and other renal cell carcinoma subtypes via immunohistochemistry and molecular studies.

\section{ACKNOWLEDGEMENTS}

The authors would like to thank Dr. Robin Bideau for providing clinical information and the radiographic images.

\section{References}

1. Argani P, Hawkins A, Griffin CA, Goldstein JD, Haas M, Beckwith JB, et al. A distinctive pediatric renal neoplasm characterized by epithelioid morphology, basement membrane production, focal HMB45 immunoreactivity, and $\mathrm{t}(6 ; 11)(\mathrm{p} 21.1 ; \mathrm{q} 12)$ chromosome translocation. Am J Pathol 2001; 158, 2089-2096.

2. Petersson F, Vanecek T, Michal M, Martignoni G, Brunelli M, Halbhuber Z, et al. A distinctive translocation carcinoma of the kidney; "rosette forming," t(6;11), HMB45positive renal tumor: a histomorphologic, immunohistochemical, ultrastructural, and molecular genetic study of 4 cases. Hum Pathol 2012; 43, 726-736.

3. Smith NE, Illei PB, Allaf M, Gonzalez N, Morris K, Hicks J, et al. t(6;11) renal cell carcinoma (RCC): expanded immunohistochemical profile emphasizing novel RCC markers and report of 10 new genetically confirmed cases. Am J Surg Pathol 2014; 38, 604-614.

4. Argani P, Yonescu R, Morsberger L, Morris K, Netto GJ, Smith N, et al. Molecular confirmation of $\mathrm{t}(6 ; 11)(\mathrm{p} 21 ; \mathrm{q} 12)$ renal cell carcinoma in archival paraffin-embedded 
material using a break-apart TFEB FISH assay expands its clinicopathologic spectrum. Am J Surg Pathol 2012; 36, 1516-1526.

5. Rao Q, Liu B, Cheng L, Zhu Y, Shi QL, Wu B, et al. Renal cell carcinomas with $\mathrm{t}(6 ; 11)(\mathrm{p} 21 ; \mathrm{q} 12)$ : A clinicopathologic study emphasizing unusual morphology, novel alpha-TFEB gene fusion point, immunobiomarkers, and ultrastructural features, as well as detection of the gene fusion by fluorescence in situ hybridization. Am J Surg Pathol 2012; $36,1327-1338$.

6. Rao Q, Zhang XM, Tu P, Xia QY, Shen Q, Zhou XJ, et al. Renal cell carcinomas with $\mathrm{t}(6 ; 11)(\mathrm{p} 21 ; \mathrm{q} 12)$ presenting with tubulocystic renal cell carcinoma-like features. Int $\mathrm{J}$ Clin Exp Pathol 2013; 6, 1452-1457.

7. Srigley JR, Delahunt B, Eble JN, Egevad L, Epstein JI, Grignon D, et al. The International Society of Urological Pathology (ISUP) Vancouver Classification of Renal Neoplasia. Am J Surg Pathol 2013; 37, 1469-1489.

8. Argani P. MiT family translocation renal cell carcinoma. Semin Diagn Pathol 2015; $32,103-113$.

9. Martignoni G, Pea M, Gobbo S, Brunelli M, Bonetti F, Segala D, et al. CathepsinK immunoreactivity distinguishes MiTF/TFE family renal translocation carcinomas from other renal carcinomas. Mod Pathol 2009; 22, 1016-1022.

10. Cholet C, Eiss D, Cohen D, Verkarre V, Helenon O. Calcified Renal Angiomyolipoma : A Case Report. Urology 2016.

11. Richmond L, Atri M, Sherman C, Sharir S. Renal cell carcinoma containing macroscopic fat on CT mimics an angiomyolipoma due to bone metaplasia without macroscopic calcification. Br J Radiol 2010; 83, e179-181. 
12. Aron M, Aydin H, Sercia L, Magi-Galluzzi C, Zhou M. Renal cell carcinomas with intratumoral fat and concomitant angiomyolipoma: potential pitfalls in staging and diagnosis. Am J Clin Pathol 2010; 134, 807-812.

13. Zhao M, Williamson SR, Yu J, Xia W, Li C, Zheng J, et al. PAX8 expression in sporadic hemangioblastoma of the kidney supports a primary renal cell lineage: implications for differential diagnosis. Hum Pathol 2013; 44, 2247-2255.

14. Doyle LA, Fletcher CD. Peripheral hemangioblastoma: clinicopathologic characterization in a series of 22 cases. Am J Surg Pathol 2014; 38, 119-127.

15. Kuroda N, Agatsuma Y, Tamura M, Martinek P, Hes O, Michal M. Sporadic renal hemangioblastoma with CA9, PAX2 and PAX8 expression: diagnostic pitfall in the differential diagnosis from clear cell renal cell carcinoma. Int J Clin Exp Pathol 2015; 8, 2131-2138.

16. Verine J. Differential diagnosis of primary benign vascular tumors and/or tumorlike lesions of the kidney: immunohistochemical stains should not be restricted to vascular and pan cytokeratin markers. Virchows Arch 2013; 462, 365-367.

17. Carney EM, Banerjee P, Ellis CL, Albadine R, Sharma R, Chaux AM, et al. PAX2(-)/PAX8(-)/inhibin A(+) immunoprofile in hemangioblastoma: A helpful combination in the differential diagnosis with metastatic clear cell renal cell carcinoma to the central nervous system. Am J Surg Pathol 2011; 35, 262-267.

18. Sancheti S, Menon S, Mukherjee S, Arun I. Clear cell renal cell carcinoma with hemangioblastoma-like features: A recently described pattern with unusual immunohistochemical profile. Indian J Pathol Microbiol 2015; 58, 354-355. 
19. Montironi R, Lopez-Beltran A, Cheng L, Galosi AB, Montorsi F, Scarpelli M.

Clear cell renal cell carcinoma (ccRCC) with hemangioblastoma-like features: a previously unreported pattern of ccRCC with possible clinical significance. Eur Urol 2014; 66, 806810.

\section{Figure Legends:}

Figure 1: (A) Computed tomography demonstrates a heterogeneously enhancing small endophytic renal mass in the right kidney (white arrow). (B) The tumor is composed of extensive sclerosis with metaplastic bone formation, adipose tissue and hyalinized, thick blood vessels (top, H\&E 100x magnification). Higher magnification (C, H\&E 200x magnification) reveals epithelial cells with clear to eosinophilic and foamy (D, H\&E 400x magnification) cytoplasm and moderate variation in nuclear size. A distinct nested arrangement with delicate vascular network, as would be expected of clear cell renal cell carcinoma, is lacking.

Figure 2: The epithelial cells label diffusely for keratin AE1/AE3 (A, 100x magnification) and PAX8 (B, 100x magnification). There is patchy reactivity for melan-A $(\mathrm{C}, 100 \mathrm{x}$ magnification) and cathepsin $\mathrm{K}(\mathrm{D}, 100 \mathrm{x}$ magnification). Positivity for HMB45 is very limited compared to melan-A, with only rare scattered cells showing a positive reaction (E, arrows, 100x magnification and inset 200x magnification). Fluorescence in situ hybridization reveals rearrangement of $T F E B$ (F, 1000x magnification), with one signal pair showing non-rearranged fused signals (white arrow) and the other signal pair being widely separated (black arrows). 


\begin{abstract}
Renal cell carcinoma with $T F E B$ rearrangement $[\mathrm{t}(6 ; 11)(\mathrm{p} 21 ; \mathrm{q} 13)]$ was initially recognized to be composed of dual populations of large cells with clear cytoplasm and small cells forming rosettes around hyaline material. With increasing awareness, however, the spectrum of described morphology has been found to be more heterogeneous. We report a 54 year-old woman who underwent partial nephrectomy for a $2.4 \mathrm{~cm}$ renal mass, composed of fibrosis, hyalinization, calcification and ossification, and a smaller component of epithelioid cells. Immunohistochemical staining revealed diffuse positivity for cytokeratin AE1/AE3 and PAX8, patchy labeling for melan-A, HMB45, and cathepsin $\mathrm{K}$, and negative caldesmon, SMA, TFE3 protein, carbonic anhydrase IX, CD10, CK7, EMA and inhibin. Fluorescence in situ hybridization confirmed rearrangement of TFEB and not TFE3. Together with one recent case in another report, our findings suggest that extensive sclerosis and ossification may be a less common recurring histology of TFEB rearrangement renal cell carcinoma.
\end{abstract}


Williamson et al - Sclerosing TFEB Renal Cell Carcinoma
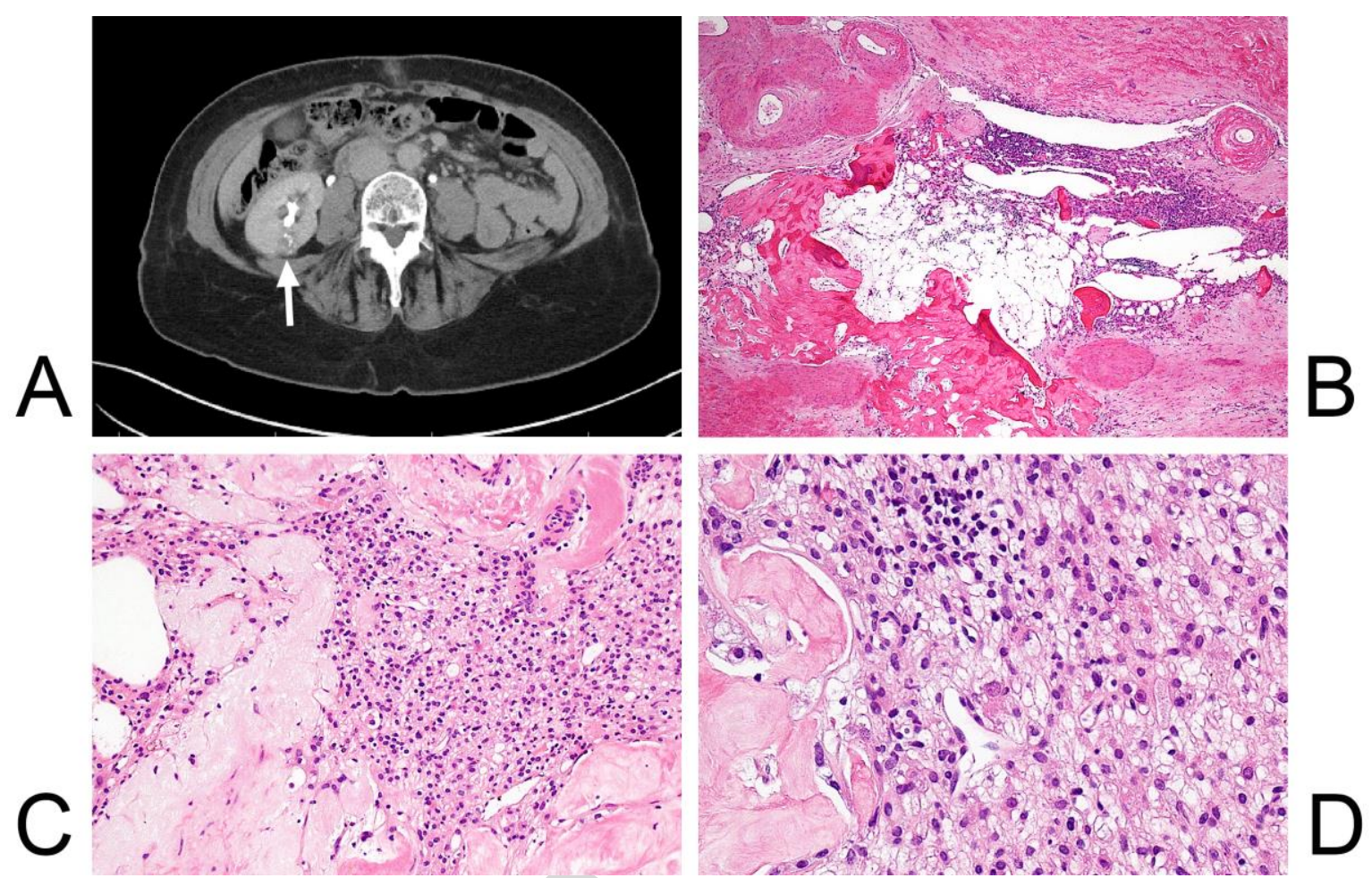

Figure 1 
Williamson et al - Sclerosing TFEB Renal Cell Carcinoma

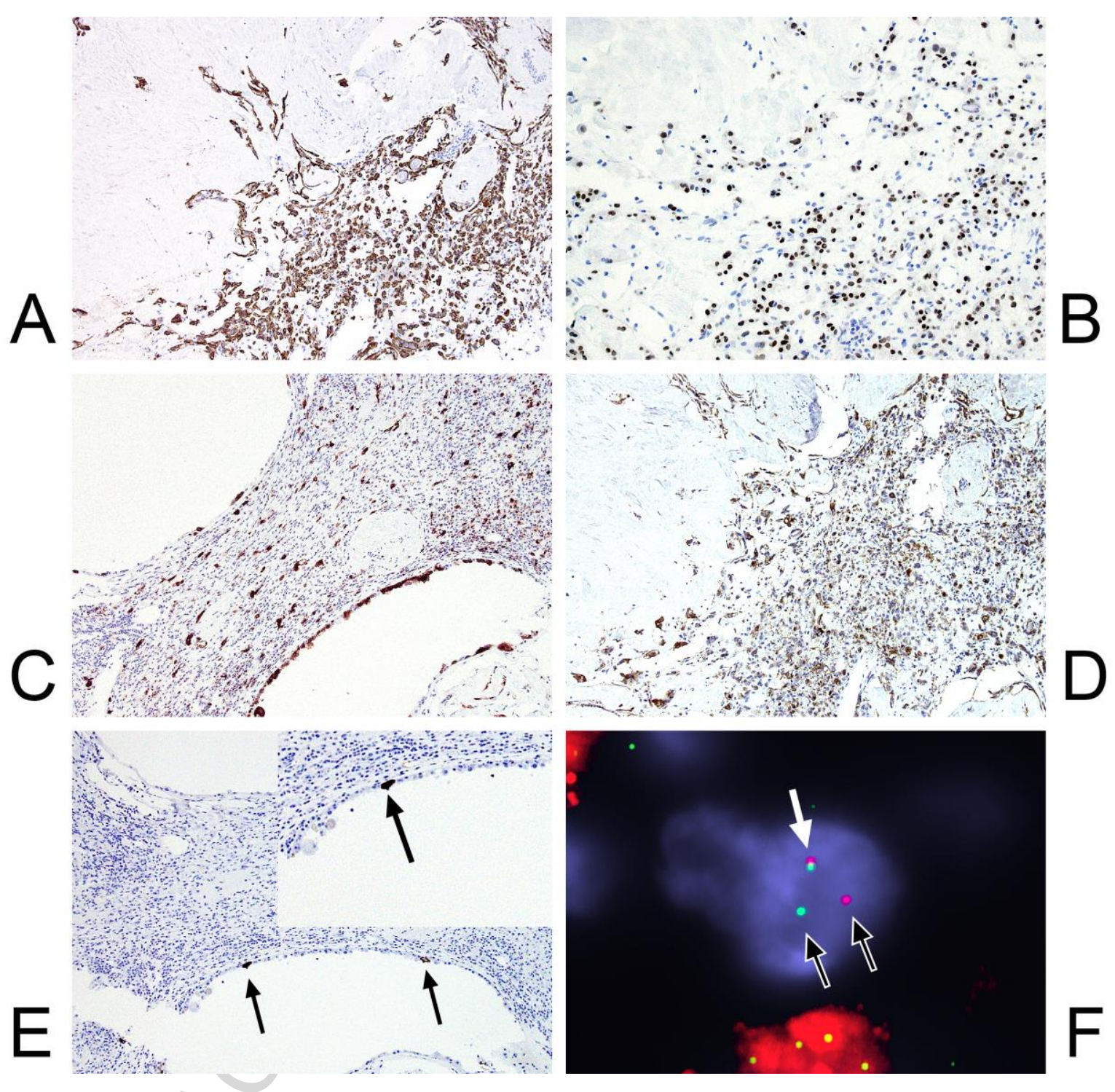

Figure 2 